\title{
STRUCTURES HIÉRARCHIQUES EN TERMINOLOGIE - L'HYPERONYMIE
}

\begin{abstract}
Kacprzak Alicja, Structures hiérarchiques en terminologie - l'hyperonymie [Hierarchic structures in terminology - hyperonymy], Studia Romanica Posnaniensia, Adam Mickiewicz University Press, Poznań, vol. XXVII: 2001, pp. 83-91, ISBN 83-232-1039-X, ISSN 0137-2475.

Along with antonymy and synonymy, hyperonymy is one of the basic semantic relations in lexis. In specialistic terminologies the status of hyperonymy seems to be exceptional since this type of vocabulary reflects the hierarchic character so typical for many fields of science. This article presents the phenomenon of hyperonymy in the language of medicine in which it does not only perform the task of ordering terminology, but is also an important factor which is a condition of creation of new terms.
\end{abstract}

D'une manière générale, les linguistes sont aujourd'hui d'accord pour considérer l'hyperonymie comme l'une des relations sémantiques fondamentales du lexique, à côté de la synonymie et de l'antonymie (citons à ce propos, par exemple, les noms de J. Lyons, 1975: 496 et de P. Lerat, 1995: 85). Il semble que le premier de ces trois phénomènes puisse avoir un statut particulier dans les terminologies qui, très souvent, témoignent d'un caractère hiérarchique, tout comme les sciences dont elles sont l'expression. En effet, ce qui décide de la structure d'un vocabulaire, c'est la structure de la culture qu'il transmet (Lyons, 1975: 499), on peut donc s'attendre à ce que la terminologie médicale reflète le réseau de dépendances existant au sein de la réalité médicale.

On définit l'hyperonymie comme: 'relation du générique au spécifique' (Lerat, 1995: 86), comme 'opposition sémantique privative' (Weinsberg, 1983: 108), comme 'implication unilatérale' (Lyons, 1975: 497). En effet, ce phénomène, à la différence de l'antonymie qui est basée sur le principe binaire, établit un rapport d'inclusion (à comparer par ex. dans K.-D. Bünting, 1989: 217) qui peut concerner un ou plusieurs termes (hyponymes) par rapport à un terme superordonné, appelé aussi hyperonyme. Ce rapport est unilatéral, ainsi si l'on considère que chat est un hyponyme de animal, on peut poser que: 
A est un chat implique $A$ est un animal,

mais non

A est un animal implique $A$ est un chat.

Le même superordonné peut avoir sous sa dépendance sémantique un ou plusieurs termes cohyponymes qui sont de même niveau, sans aucun rapport d'inclusion. L'hyperonyme animal inclut un grand nombre de cohyponymes, comme chat, chien, éléphant, vache, etc. Les cohyponymes sont compatibles entre eux, c'est-à-dire que tout en étant distincts, ils appartiennent à la même classe.

Sans doute, le phénomène de l'hyperonymie reflète-t-il en quelque sens le réseau de la pensée humaine, organisatrice du monde qui l'entoure. De l'autre côté, ce phénomène a un pouvoir fondateur qui se retrouve à la base d'un nombre considérable de dénominations. C'est aussi le cas de la langue médicale qui, à cause de ses besoins spécifiques d'ordre et de clarté, exploite très souvent ce modèle de motivation. Évidemment, l'hyperonymie fonctionne avant tout comme un phénomène logique permettant la structuration de champs notionnels, ainsi le terme superordonné maladie inclut un grand nombre de noms de maladies, par ex. grippe. varicelle, diabète, tuberculose, anémie, etc. A son tour, le terme anémie peut constituer un hyperonyme pour les termes dénotant différents types d'anémie, par ex. anémie achrestique, anémie agastrique, anémie globulaire, anémie carentielle, anémie hémolytique. etc. Chaque terme cohyponyme est inclus par l'hyperonyme et, d'une certaine manière, il reste en opposition par rapport à tous les autres cohyponymes. Cette relation rappelle donc celle de l'antonymie (complémentaire), avec cependant deux différences fondamentales:

1) l'antonymie envisage l'opposition polaire de deux termes (par ex. hypotension et hypertension), par contre l'hyperonymie - les oppositions mutuelles de plusieurs termes cohyponymes (par ex. veine temporale profonde, veine temporale movenne. veine temporale superficielle:

2) dans le rapport de complémentarité, la négation du terme $A$ entraîne l'affirmation du terme $B$, dans l'hyperonymie, la négation du terme $A$ implique l'affirmation virtuelle de l'un de termes cohyponymes, donc si $A$ n'est pas, il y a peut-être $B$, ou $C$, ou $D$, etc.

Dans la langue médicale, ce modèle sémantique a servi à la formation d'un grand nombre de termes qui, subordonnés à un terme générique s'opposent mutuellement entre eux. Les types de fondements de ces termes sont variés, il n'en reste pas moins que, d'une manière supplémentaire, ils sont motivés par l'appartenance à la même structure paradigmatique.

Les séries d'hyponymes apparaissent au sein de tous les champs notionnels du vocabulaire médical et surtout dans les groupes suivants:

- les dénominations de maladies. Le terme superordonné est un nom à caractère générique dénotant une maladie dont les variantes sont exprimées par les noms formés du terme générique accompagné d'un adjectif ou d'un complément 
précisant le type de maladie, par ex. diabète et diabète sucré, diabète bronzé. diabète gras, diabète maigre. diabète fruste, diabète des femmes à barbe: gale et gale bédouine, gale du ciment, gate filarienne, gale norvégienne, gale aponévrotique:

- les dénominations de syndromes, symptômes, etc., par ex. ictère et ictère acholurique, ictère bénin précoce. ictère à bilirubine conjuguée, ictère catarrhal. ictère hépatique. etc.; cœur (au sens de symptômes liés au cœur) et cœur croisé. cœur irritable, cœur forcé, cœur biloculaire, cœur de bœuf:

- les dénominations anatomiques, par ex. artère et artère tibiale, artère tvmpanique, artère vertébrale, artère sous-orbitraire, artère apendiculaire; $\underline{\text { os }}$ et $\underline{\text { os }}$ iliaque, os cuboïde, os crochu, os frontal, os éthmoïde, os pisiforme. os semilunaire. etc.

En guise d'exemple, voici la représentation de la série hyperonymique du terme superordonné: anémie (selon le Dictionnaire des termes de médecine de M. Garnier et V. Delamare, Maloine, 1989):

1) anémie achrestique (syn. maladie de Wilkinson);

2) anémie d'Addison (syn. a. de Biermer, a. idiopathique, a. hyperchrome mégalocytique, a. pernicieuse progressive);

3) anémie agastrique;

4) anémie aiguë curable du nouveau-né (syn. anémie aiguë du nouveau-né, type Lelong-Joseph);

5) anémie aiguë fébrile (syn. anémie hémolytique fébrile, anémie hémolytique aiguë, anémie de Brill, anémie de Lederer, anémie de Lederer-Brill);

6) anémie aplastique (syn. anémie agénérative, anémie médullaire);

7) anémie arégénérative (syn. anémie aplastique);

8) anémie arégénérative chronique et congénitale (syn. anémie de Blackfan-Diamond);

9) anémie de Biermer (syn. anémie d'Addison, anémie hyperchrome mégalocytique, anémie idiopathique, anémie pernicieuse progressive);

10) anémie de Brill (syn. anémie aiguë fébrile, anémie hémolytique fébrile, anémie hémolytique aiguë, anémie de Lederer, anémie ou maladie de Lederer-Brill);

11) anémie ou maladie de Lederer-Brill, anémie hémolytique aiguë);

12) anémie des briquetiers (syn. ankylostomasie);

13) anémie carentielle (syn. anémie nutritionnelle, anémie de famine, anémie lévurocurable, anémie macrocytaire de nutrition, anémie tropicale, anémie de Lucy Wills, anémie de Lucy, anémie de Wills);

14) anémie de Cooley (syn. maladie de Cooley, syndrome de Cooley, anémie méditerranéenne);

15) anémie cryptogénétique (ou cryptogénique);

16) anémie drépanocytaire (syn. drépanocytose, siklémie, hémoglobinose $S$, maladie de Herrick, syndrome de Dresbach, anémie à hématies falciformes); 
17) anémie elliptocytique, (syn. ovalocytose, anémie ovalocytique, elliptocytose, maladie de Dresbach);

18) anémie enzymoprive (syn. anémie par enzymopathie);

19) anémie érythroblastique;

20) anémie érythrodysgénésique;

21) anémie essentielle (syn. anémie essentielle des jeunes filles, chlorose);

22) anémie familiale perniciosiforme (syn anémie de Fanconi);

23) anémie de famine (syn. anémie de Wills, a. de Lucy Wills, a. de Lucy, anémie lévurocurable, anémie macrocytaire de nutrition, anémie tropicale);

24) anémie ferriprive (syn. anémie sydéropénique, anémie hypochrome hyposidérémique);

25) anémie des géophages;

26) anémie globulaire (syn. anémie hyperchrome, anémie hémoglobinique);

27) anémie grave érythroblastique du nouveau-né;

28) anémie de Hayem-Faber;

29) anémie à hématies falciformes (syn. anémie drépanocytaire, syn. drépanocytose, siklémie, hémoglobinose $S$, maladie de Herrick, syndrome de Dresbach);

30) anémie hémoglobinique (syn. anếmie hypochrome);

31) anémie hémolytique;

32) anémie hémolytique aiguë (syn. anémie de Brill, anémie aiguë fébrile, anémie hémolytique fébrile, anémie de Lederer, anémie ou maladie de Lederer-Brill);

33) anémie hémolytique auto-immune (ou à auto-anticorps);

34) anémie hémolytique enzymoprive (syn. anémie hémolytique par enzymopathie, érythro-enzymopathie);

35) anémie hémolytique héréditaire non-sphérocytaire (syn. maladie de Thompson);

36) anémie hémolytique immunologique;

37) anémie hémolytique micro-angiopathique;

38) anémie hémolytique périnatale;

39) anémie hyperchrome (syn. anémie hyperchromique);

40) anémie hypochrome hypersidérémique;

41) anémie hypochrome (syn. anémie hypochromique, anémie hémoglobinique);

42) anémie hypochrome hyposidérémique (syn. anémie sidéropénique, anémie ferriprive);

43) anémie hypoplastique du petit enfant (syn. a. h. congénitale, a. h. idiopathique, a. h. permanente);

44) anémie hypoplastique avec pouces anormaux;

45) anémie idiopathique (syn. anémie de Biermer, anémie d'Addison, anémie hyperchrome mégalocytique, anémie pernicieuse progressive); 
46) anémie d'Imerslund-Najman-Gräsbeck (syn. maladie d'I.-N.-G., anémie mégaloblastique par malabsorption sélective de la vitamine $\mathrm{B}_{12}$ );

47) anémie infantile pseudo-leucémique (syn. a. i. splenique);

48) anémie isochrome (syn. anémie normochrome, anémie orthochrome);

49) anémie leuco-érythroblastique (syn. splénomégalie myéloide, anémie avec myélémie et splénomégalie, anémie splénique érythromyéloïde, anémie splénique myéloïde, érythroblastose chronique de l'adulte, leucémie ostéosclérotique, leuco-érythroblastose, maladie érythroblastique de l'adulte, métaphasie érythromyéloïde hépatosplénique avec myélofibrose, myélose aleucémique mégacaryocytaire, panmyélose splénomégalique chronique, splénomégalie chronique avec anémie et myélémie, splénomégalie érythroblastique ou érythromyéloïde, splénomégalie myéloïde mégacaryocytaire, splénomégalie myéloide avec myélocythémie, splénomégalie avec sclérose de la moelle osseuse);

50) anémie macrocytaire (ou macrocytique, anémie mégalocytaire);

51) anémie maligne intermédiaire;

52) anémie méditerranéenne;

53) anémie mégalocytaire (ou mégalocytique);

54) anémie mégaloblastique;

55) anémie microcytaire (ou microcytique);

56) anémie microcytique drépanocytaire de Silvestroni et Bianco (syn. anémie microdrépanocytaire, thalasso-drépanocytose);

57) anémie des mineurs (syn. ankylostomasie);

58) anémie avec myélémie et splénomégalie (synonymes comme anémie leucoérythroblastique);

59) anémie nutritionnelle (syn. anémie carentielle, anémie de famine, anémie lévuro-curable, anémie macrocytaire de nutrition, anêmie tropicale, anémie de Lucy Wills);

60) anémie orthochrome (syn. anémie isochrome, anémie normochrome);

61) anémie ostéosclérotique (ou ostéoscléreuse);

62) anémie ovalocytique (syn. ovalocytose);

63) anémie parabiermérienne;

64) anémie pernicieuse (syn. anẻmie d'Addison, a. de Biermer, a. idiopathique, a. hyperchrome mégalocytique, a. pernicieuse progressive);

65) anémie pernicieuse gravidique;

66) anémie pernicieuse juvénile;

67) anémie pernicieuse juvénile type I de Hoffbrand;

68) anémie pernicieuse juvénile type II de Hoffbrand;

69) anémie du Pérou;

70) anémie phagocytaire (syn. syndrome de Malin);

71) anémie plastique;

72) anémie préleucoblastique;

73) anémie protéiprive; 
74) anémie réfractaire;

75) anémie réfractaire avec myéloblastose partielle (syn. insuffisance médullaire qualitative avec myéloblastose partielle, leucémie oligoblastique);

76) anêmie sidéro-achrestique (ou sidéroblasique acquise idiopathique);

77) anémie sidéro-achrestique (ou sidéroblastique héréditaire, syn. anémie ou syndrome de Rundlers et Falls);

78) anémie sidéropénique;

79) anémie sphérocytaire;

80) anémie tropicale (syn. anémie de Lucy Wills, anémie carentielle, anémie nutritionnelle, anémie de famine, anémie lévurocurable, anémie macrocytaire de nutrition, anémie de Lucy Wills, anémie de Lucy, anémie de Wills);

La série d'hyponymes du terme superordonné anémie contient 80 termes, dont plusieurs possèdent des synonymes. Du point de vue formel, les termes cohyponymes se composent du mot anémie accompagné par une ou plusieurs adjectifs épithètes, ou bien par un complément déterminatif, ou bien par une structure mixte: épithète et complément en même temps. L'analyse détaillée permet d'en distinguer des variantes dans lesquelles le terme superordonné est accompagné par:

- un adjectif simple, par ex. anémie pernicieuse. anémie plastique:

- deux ou plusieurs adjectifs simples, par ex. anémie aiguë fébrile. anêmie arégénérative chronique et congénitale:

- un adjectif simple et un adjectif composé, par ex. anémie hémolvtique immuno-allergique:

- un adjectif composé, par ex. anémie sidéro-achrestique:

- deux adjectifs composés, par ex. anémie hyperchrome hypersidérémique:

- un ou deux adjectifs et un complément déterminatif introduit par une préposition, par ex. anémie essentielle des ieunes filles, anémie aiguë curable du nouveau-né:

- un complément déterminatif introduit par une préposition, par ex. anẻmie des briquetiers:

- un éponyme, par ex. anémie de Brill.

La structure sémantique des formations citées comporte le nom de phénomène anémie qui est spécifié par le deuxième terme (épithète ou complêment). On analyse le rapport qui existe entre le terme principal et le mot de base du second terme. Parmi eux, quelques-uns sont les plus fréquents:

- relation (phénomène/cause) - le phénomène représenté par le terme principal est causé par ce qui est dénommé par le mot de base de l'adjectif épithète ou bien par le complément déterminatif. par ex. anémie nutritionnelle 'anémie 
provoquée par l'absence de nutrition', anémie hémolvtique 'anémie due à l'hémolyse', anémie hémoglobinique 'anémie par manque d'hémoglobine', anémie de famine 'anémie causée par la famine';

- relation (phénomène/objet) - le phénomène représenté par le terme principal est caractérisé par la présence ou, plus souvent, par l'absence d'un objet, par ex. anémie megalocvtaire 'anémie caractérisée par la présence de mégalocytes', anémie enzymoprive 'anémie caractérisée par le manque d'enzymes', anémie protéiprive 'anémie caractérisée par le manque de protéines, anémie ferriprive 'anémie caractérisée par le manque de fer';

- relation (phénomène/lieu) - le phénomène représenté par le terme principal est caractérisé par sa localisation spatiale, par ex. anémie tropicale 'anémie des pays tropicaux', anémie méditerranéenne 'anémie que l'on rencontre dans les pays de la Méditerranée', anémie du Pérou 'anémie propre à certaines vallées $d u$ Pérou';

- relation (phénomène/affecté) - le phénomène est caractérisé par son bénéficiaire ( c'est-à-dire celui qui en est affecté), avec l'insistance sur son âge, par ex. anémie (aiguë curable) du nouveau-né. anémie infantile (pseudo-leucémique). anémie (essentielle) des jeunes filles, ou avec l'insistance sur sa profession, par ex. anémie des briquetiers, anémie des mineurs. ou encore sur une autre caractéristique, par ex. anémie des géophages (géophage 'celui qui mange de la terre'). Notons un type rare de formation éponymique provenant du nom du patient: anémie de Lucy Wills:

- relation de similarité - le phénomène est caractérisé par sa ressemblance à ce qui est dénoté par le mot de base, par ex. anémie parabiermérienne 'anémie qui ressemble à l'anémie de Biermer';

- relation (phénomène/temps) - le phénomène est caractérisé par la localisation temporelle évoquée par le second terme, par ex. anémie (hémolytique) périnatale 'anémie de l'enfant dans la période avant ou après sa naissance'.

Dans plusieurs termes les relations sont plus complexes, du fait que le mot principal est accompagné par deux ou même plusieurs épithètes ou compléments dont les sens s'accumulent, comme dans anémie hémolytique enzymoprive où l'on retrouve le rapport (phénomène/cause) et (phénomène/objet).

L'analyse de la série d'hyponymes du terme superordonné anémie a permis de voir qu'à côté de la relation d'hypero-/ hyponymie, il y apparaît aussi d'autres relations sémantiques entre les termes, notamment celle de la synonymie et de l'antonymie.

D'abord, il faut souligner le fait que certains parmi les cohyponymes constituent à leur tour des termes superordonnés par rapport aux autres termes, par ex. anémie plastique est un superordonné par rapport à anémie plastique orthoplastique et à anémie plastique métaplastique. Dans le cas de anémie pernicieuse. la situation est encore plus compliquée, car ce terme-ci est un superordonné par rapport à anémie pernicieuse gravidique et anémie pernicieuse iuvénile; à son tour, ce dernier possède deux cohyponymes: anémie pernicieuse iuvénile type I de Hoffbrand et anémie pernicieuse iuvénile type II de Hoffbrand. 
Ainsi, assiste-t-on au phénomène de l'hyperonymie complexe, 'à tiroirs', car tous les termes énumérés sont subordonnés par rapport à anémie.

Ensuite, comme nous l'avons déjà remarqué, plusieurs termes possèdent des synonymes, dans le cas de anémie leuco-érythroblastique on en relève même 17 . Leurs formes peuvent être variées: plusieurs d'entre eux sont construits autour d'un autre substantif à valeur générique, par ex. insuffisance, maladie, leucémie, comme dans insuffisance médullaire qualitative avec muéloblastose partielle, maladie d'Ecklin. leucémie oligoblastique. Cependant, la persistance des termes construits sur le nom anémie, à côté des termes synonymiques, semble constituer une preuve du caractère organisateur de l'hyperonymie au sein de la terminologie médicale: le mot anémie apparaissant dans les dénominations permet une organisation claire et univoque de ce groupe de termes. De cette façon, la structuration de la science se trouve reflétée dans la structuration du vocabulaire.

Enfin, quelques-uns parmi les cohyponymes se voient entre eux en relation supplémentaire d'antonymie, comme dans le cas de anémie microcvtaire et anémie macrocytaire.

En reprenant l'idée d'A. Weinsberg (1983: 108) selon laquelle l'hyperonymie est basée sur une opposition sémantique privative, il faut admettre que chaque signe dénote un ensemble de traits propres pour décrire un objet ou un phénomène. Le rapport de l'hyperonymie consiste dans le fait que tous les termes de la série comportent les mêmes traits que leur hyperonyme, mais que chaque hyponyme comporte en plus encore un (ou plusieurs) trait supplémentaire. Il en résulte que l'hyperonyme est un signe dont le sens est le plus vaste mais le plus pauvre en même temps, par contre l'hyponyme a le sens plus étroit mais plus riche. En ce sens, l'hyperonyme et l'hyponyme restent en opposition, dans laquelle le premier d'entre eux constitue un élément non-marqué et le deuxième est un élément marqué.

Pour conclure, il convient de souligner la valeur du phénomène de l'hyperonymie dans la structuration de la terminologie médicale. La liaison entre les formes linguistiques et les aspects de la réalité extralinguistique est évidente. Cependant, insistons aussi sur le fait que c'est le lexique qui hérite des hiérarchies existant dans le monde réel et non pas le contraire; comme le dit P. Lerat dans Les langues spécialisées (1995: 152): 'la soumission au réel des langues dissipe des illusions sur la modélisation des connaissances, tant ordinaires que savantes'.

\section{BIBLIOGRAPHIE}

Búnting K.-D. (1989), Wstęp do lingwistyki, Państwowe Wydawnictwo Naukowe, Warszawa. Dubois T. et alt. (1994), Dictionnaire de linguistique et des sciences du langage, Larousse, Paris. Garnier M., et Del a mare V. (1989), Dictionnaire des termes de médecine, Maloine, Paris. Kleiber G., T a mba I. (1990), L'hyponymie revisitée: inclusion et hiérarchie, Langages no 98. Lerat P. (1990), L'hyperonymie dans la structuration des terminologies, Langages, no 98. 
Lerat P. (1995), Les langues spécialisées, P.U.F., Paris.

Lyons J. (1975), Wstęp do językoznawstwa, Państwowe Wydawnictwo Naukowe, Warszawa.

Pi coche J. (1992), Précis de lexicologie française, Nathan.

Weinsberg A. (1983), Jezzykoznawstwo ogólne, Państwowe Wydawnictwo Naukowe, Warszawa. 